\title{
Devenir des œufs et kystes de parasites au cours d'un cycle d'épuration de la station Cherguia à Tunis
}

\author{
Fate of parasites eggs and cysts in the course of waste water treatment \\ cycle of the Cherguia station in Tunis
}

par Zoubeir Alouini

Laboratoire de parasitologie des eaux usées, Institut National de Recherche en Génie rural, Eaux et Forêts

In Tunisia particular attention is accorded to the sanitation aspects, when treated waste water is reused for agricultural purposes. The current water quality standard requires an average number inferior or equal to one intestinal helminth egg per litre of treated waste water. Information concerning parasitic risk is however rather limited in Tunisia. In this context researches for protozoa cysts and helminth eggs were conducted on raw waste water, water after primary decantation, treated waste water, primary and secondary sewage sludge from the biological treatment plant in Cherguia.

The identified protozoa include Amebae and Flagellates. Among the Amebae, Entamoeba coli and Entamoeba histolytica cysts are detected, among the Flagellates, Giardia spp cysts are found. As a general rule, this later predominates. Among the helminths, Nematods and cestods are observed. The average number of helminth eggs is markedly lower. For in inlet water, all analysed samples contained protozoa cysts particulary Giardia cysts in mean concentration of 2.1. $10^{2}$ cysts $t^{-1}$. In lower frequency we found helminth eggs in mean concentration from 4 to 2,6. $10^{1}$ eggs $t^{1}$.

Through the treatment chain the greater part of eggs were eliminated during the primary decantation. The efficiency of elimination is about $30 \%$ for cysts and $63 \%$ for eggs.

In treated waste water the efficiency of the cysts elimination is about $97 \%$ and $100 \%$ of the samples were exempt from helminth eggs. The use of activated sludge is thus demonstrated, as very efficient for removal of parasitic elements.

\section{INTRODUCTION}

En Tunisie, l'introduction des eaux usées comme ressource complémentaire dans le plan national de la valorisation des ressources en eau constitue un apport intéressant pour l'agriculture qui souffre d'un déficit hydrique. Or cette pratique impose une appréciation des risques sanitaires encourus pour l'homme et le bétail. La norme tunisienne de qualité en vigueur (NT 106.03, 1989) fixe un nombre moyen inférieur ou égal à 1 œuf d'helminthes intestinaux par litre d'eau usée traitée. Or certains travaux [1,2] montrent que l'agent pathogène le plus communément identifié aux USA dans les épidémies véhiculées par l'eau est un protozoaire (Giardia). Eckert [3] indique une prévalence de 3,6\% en Suisse.

En Tunisie la prévalence de la protozoose diffère en fonction des régions. Kennou [4] indique une fréquence de Giardiose de $20,1 \%$ au sud du pays (vers la région de Gabès) et $35,6 \%$ dans la région de Tunis. Il existe dans la population des malades et des porteurs sains excrétant dans leurs selles les formes de contamination (kystes). Ces kystes au même titre que les œufs d'helminthes sont présents dans les eaux usées et les différents produits résultant de leur épuration [6]. Par ailleurs certaines de ces formes en raison de leur grande résistance ou de leur petite taille, pourraient constituer un marqueur d'efficacité de traitement. Dans cette optique, il nous paraît intéressant d'étudier le devenir des œufs et kystes de parasites au cours d'un cycle d'épuration conduit en Tunisie.

\section{II — MATÉRIEL ET MÉTHODES}

Les prélèvements sont effectués à la station d'épuration de la Cherguia située à Tunis-Nord, station de type biologique traitant la pollution urbaine d'environ 600000 habitants. Sa capacité de traitement est de $60000 \mathrm{~m}^{3}$ d'eaux usées par jour. La chaîne d'épuration comporte un prétraitement assuré par un dégrilleur manuel, un dessableur aéré de volume $240 \mathrm{~m}^{3}$ avec un temps de séjour de 6 minutes et un déshuileur circulaire de volume $550 \mathrm{~m}^{3}$ avec un temps de séjour de 13 minutes. Le traitement primaire est assuré par 4 décan- 
teurs rectangulaires de volume total $4320 \mathrm{~m}^{3}$ et un temps de séjour de 2 heures. Un réacteur à boues activées à mélange complet, est constitué de 8 bassins totalisant un volume de $9000 \mathrm{~m}^{3}$ avec un temps de rétention de 3 heures. La décantation secondaire est assurée par 4 bassins circulaires. Le traitement des boues est réalisé par deux digesteurs primaires et un secondaire de volume $4000 \mathrm{~m}^{3}$ chacun. Le séchage des boues digérées est assuré par 200 lits de séchage sur sable d'une superficie globale de $32000 \mathrm{~m}^{2}$. Le temps de séjour dans la station est d'environ 6 heures.

Les analyses ont été effectuées :

- sur les eaux usées brutes ( $1^{\circ}$ point).

- sur les eaux traitées à différents niveaux d'épuration : en sortie de décanteur primaire ( $2^{\circ}$ point) et de station $\left(5^{\circ}\right.$ point $)$, au niveau des boues primaires $\left(3^{\circ}\right.$ point) et secondaires $\left(4^{\circ}\right.$ point $)$ - (voir figure 1$)$.

Les prélèvements sont effectués à heure fixe et jour fixe, deux fois par semaine, à partir du mois de janvier jusqu'au mois de juin 1994. Le volume des échantillons d'eau usée analysé est de 5 litres. Pour les boues, le volume des échantillons est de $1 \mathrm{~kg}$ à partir duquel sont réalisés des souséchantillons de $100 \mathrm{~g}[6,7]$.

\section{III — MÉTHODES}

Nous avons opté pour la technique proposée dans le rapport de l'OMS $N^{\circ} 778$ [8]. Cette méthode de concentration parasitaire précédemment décrite [5] repose sur la décantation de l'échantillon pendant 24 heures. Le sédiment récupéré est centrifugé pendant 15 minutes à $1000 \mathrm{~g}$. Le culot obtenu est mélangé à un volume équivalent de tampon acéto-acétique $\mathrm{pH} 4,5$. On ajoute par la suite un volume d'éther équivalent au double du volume de la solution obtenue puis on agite pendant 10 minutes. L'ensemble est centrifugé par la suite à $1000 \mathrm{~g}$ pendant 6 minutes. Le sédiment obtenu est remis en suspension avec environ $5 \mathrm{ml}$ d'une solution de sulfate de zinc $(33 \%$, de densité 1,18$)$. Le volume $\mathrm{V}$ du produit est mesuré. Un volume $\mathrm{P}$ de $0,3 \mathrm{ml}$ est prélevé en surface après flottation pour observation microscopique.

Le nombre total d'oeufs ou kystes par litre $(\mathrm{N})$ présent dans la prise d'essai originelle est déterminé comme suit :

$$
\mathrm{N}=\frac{\mathrm{X} \cdot \mathrm{V}}{\mathrm{P} \cdot \mathrm{S}}
$$

$X$ : Nombre d'œufs (ou kystes),

P : Volume du produit observé sous microscope,

$\mathrm{V}$ : Volume du produit final,

S : Volume de l'échantillon de départ.

Pour les échantillons de boues, après décantation, les souséchantillons de $100 \mathrm{~g}$ récupérés sur le sédiment sont centrifugés à $1000 \mathrm{~g}$ pendant 6 minutes après le traitement diphasique : tampon acéto-acétique $\mathrm{pH} 4,5$ et éther.

Cette méthode d'enrichissement est la plus polyvalente, le $\mathrm{pH} 4,5$ étant le plus favorable pour concentrer l'ensemble des éléments parasitaires (œufs, kystes), la plus favorable aussi pour leur conservation (meilleure identification possible).

\section{IV — RÉSULTATS ET DISCUSSION}

En eau brute environ $91 \%$ des échantillons (voir fig. 2) contiennent des kystes de protozoaires, alors qu'après la décantation primaire $83 \%$ des échantillons d'eau sont positifs. En eau épurée seulement $14 \%$ des échantillons renferment des kystes.

En ce qui concerne les œufs d'Helminthes, $34 \%$ des échantillons d'eau brute sont positifs. Après la décantation primaire on constate que seulement $26 \%$ des échantillons contiennent des œufs. Toutefois on enregistre une absence totale d'échantillons positifs en œufs d'helminthes dans les eaux épurées à la sortie de la station.

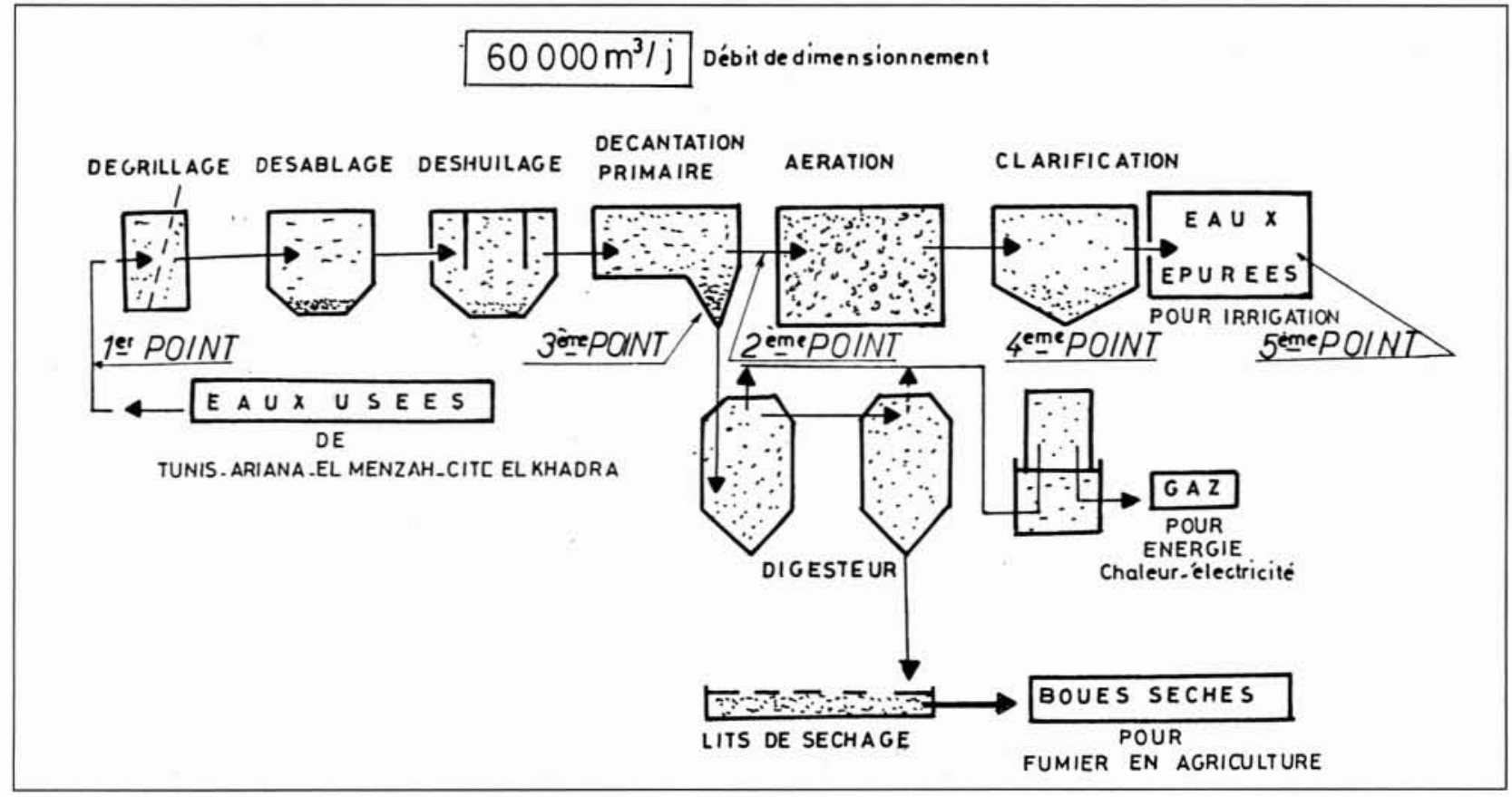

1. Station d'épuration de Cherguia -Tunis. 
Dans les boues primaires et secondaires respectivement $94 \%$ et $91 \%$ des échantillons contiennent des kystes de protozoaires. Alors que pour les æufs d'helminthes seulement $34 \%$ des échantillons de boues primaires sont positifs et $29 \%$ pour les boues secondaires.

Chez les protozoaires on rencontre les Amibes et les Flagellés. Au niveau des Helminthes ce sont les Nématodes et les Cestodes qui sont observés en accord avec les observations de Stien et Schwartzbrod [9].

Parmi les amibes sont retrouvés les kystes d'Entamoeba coli et Entamoeba histolytica, chez les Flagellés des kystes de Giardia spp.

Chez les nématodes sont observés des œufs d'Ascaris et d'oxyure, chez les cestodes des œufs d'Hymenolepis nana.

\section{- Echantillons d'eaux usées :}

Le nombre moyen de kystes de protozoaires dans les eaux usées tourne autour de 5,2.10 $10^{2} \mathrm{l}^{-1}$. Le kyste de Giardia spp prédomine avec une moyenne d'environ 2,1. $10^{2}$ kystes $\mathrm{l}^{-1}$. Cela est en accord avec les fréquences de parasitoses intestinales avancées par Kennou [4]. Dans la région de Tunis, la Giardiose présente une fréquence de 35,61\% alors que pour Entamoeba histolytica la fréquence rapportée est de $3,56 \%$.

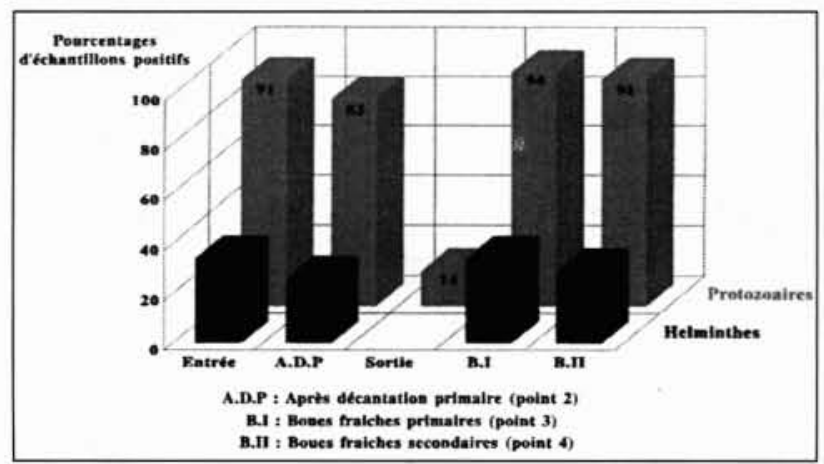

2. Pourcentages d'échantillons positifs en éléments parasitaires.
Les valeurs observées du kyste de Giardia en eau d'entrée sont voisines de celles rapportées par Schwartzbrod et al [6] avec $3,2.10^{1}$ à $2,1.10^{4}$ kystes $\mathrm{I}^{-1}$, par Panicker et al [10]

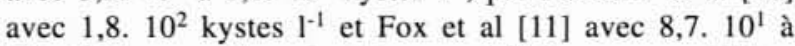
5,2. $10^{2}$ kystes $1^{-1}$, mais elles sont inférieures aux chiffres donnés par Akin et al [12] avec $8.10^{4}$ kystes $\mathrm{I}^{-1}$ ou Jakubowski et al [13] avec $9,6 \cdot 10^{3}$ à $2,4 \cdot 10^{5}$ kystes $1^{-1}$. Le nombre moyen des œufs d'Helminthes est nettement plus faible et varie selon les saisons et l'origine de l'eau. La moyenne enregistrée en eau d'entrée est de l'ordre de 3,8. $10^{1}$ œufs $1^{-1}$ (voir tableau 1).

Les œufs d'ascaris et d'oxyure semblent être relativement plus fréquents que les œufs d'Hymenolepis nana. Cela reste cependant très variable dans les différentes communes et en fonction du mode de contamination. Dans la région de Tunis, Ben Rachid [14] rapporte une fréquence de $22 \%$ pour l'Ascaridiose. Alors que pour hymenolepis nana la fréquence est de $12,5 \%$. Cette prévalence des parasitoses intestinales varie selon les auteurs. En effet les statistiques effectuées à l'Institut Pasteur à Tunis de 1976 à 1982 [4] montre une fréquence de $3,9 \%$ pour l'ascaridiose et $6,2 \%$ pour Hymenolepis nana.

L'oxyurose en Tunisie présente généralement une incidence marquée surtout en milieu scolaire tant dans les régions rurales que dans celle de Tunis [4].

- Echantillons de Boues :

les moyennes enregistrées dans les boues fraîches primaires et secondaires sont respectivement de $11,3 \cdot 10^{3}$ et $8,1.10^{3}$ kystes $100 \mathrm{~g}^{-1}$ pour les protozoaires. Alors que pour les œufs d'Helminthes elles sont relativement plus faibles avec 9,4.102 œufs $100 \mathrm{~g}^{-1}$ pour les boues primaires et $2.10^{2} 100 \mathrm{~g}^{-1}$ pour les boues secondaires. D'une façon générale le kyste de Giardia spp apparaît comme le plus fréquent avec une moyenne qui varie de 4,3 à $5,1.10^{3}$ kystes $100 \mathrm{~g}^{-1}$. Pepin et al [15] retrouvent de $1,2.10^{3}$ à $8,5.10^{4}$ kystes de Giardia $100 \mathrm{~g}^{-1}$ de matière organique dans les boues fraîches. Schwartzbrod et al [6] rapportent que dans les boues primaires la moyenne de Giardia varie de $1,6.10^{2}$ à $1,9.10^{2}$ kystes $100 \mathrm{~g}^{-1}$, alors que dans les boues semi-déshydratées elle descend à 5 kystes $100 \mathrm{~g}^{-1}$. Dans les boues digérées, Fox et al [11] ne retrouvent

\begin{tabular}{|l|c|c|c|c|c|}
\hline Station Cherguia & Entrée & A.D.P. & Sortie & BI (100 g-1) & BII (100 g-1) \\
\hline Giardia spp & $2,110^{2}$ & $1,910^{2}$ & 4,3 & $5,110^{3}$ & $4,310^{3}$ \\
Entamoeba histolytica & $0,810^{2}$ & $0,410^{2}$ & 4,3 & $2,310^{3}$ & $0,710^{3}$ \\
Entamoeba coli & $2,310^{2}$ & $1,310^{2}$ & 5,7 & $3,910^{3}$ & $3,110^{3}$ \\
\hline Total de protozoaires & $5,210^{2}$ & $3,610^{2}$ & 14,3 & $11,310^{3}$ & $8,110^{3}$ \\
Ecart type & $1,410^{2}$ & $1,210^{2}$ & 19,7 & $4,310^{3}$ & $2,210^{3}$ \\
\hline Ascaris & 26,4 & 10,4 & - & $6,710^{2}$ & $1,810^{2}$ \\
Oxyure & 7,7 & 2,6 & - & $0,710^{2}$ & $0,210^{2}$ \\
\hline Hymenolepis nana & 4,0 & 1,0 & - & $2,010^{2}$ & - \\
Total d'helminthes & 38,1 & 14,0 & - & $2,410^{2}$ & $2,010^{2}$ \\
Ecart type & 36,18 & 22,3 & - & $3,310^{2}$ & $1,310^{2}$ \\
\hline
\end{tabular}

Tableau 1 : Concentrations par litre en oufs d'Helminthes et kystes de protozoaires à différents niveaux d'épuration. A.D.P. : Après décantation primaire (point 2). ; B.I : Boues primaires (point 3).; B.II : Boues fraîches secondaires (point 4). 
aucun kyste de Giardia. Enfin Pepin et al [15] constatent que si dans les boues ayant subi un traitement thermique les kystes de Giardia ont disparu, par contre des boues traitées à la chaux et au chlorure ferrique contiennent encore $4.10^{1}$ kystes $100 \mathrm{~g}^{-1}$.

\section{$\mathrm{V} \square$ CONCLUSION - DISCUSSION}

Le devenir des éléments parasitaires au cours d'un cycle d'épuration est représenté sur le schéma 1.

Le taux d'élimination de la charge parasitaire de la station de la Cherguia est de l'ordre de $97 \%$. Les ouvrages des bassins d'activation et de la décantation secondaire interviennent dans l'abattement des éléments parasitaires. Ils achèvent l'élimination des œufs qui échappent à la décantation primaire. En effet on enregistre après la décantation primaire un taux d'élimination des œufs d'Helminthes de l'ordre de $63 \%$, alors que ce dernier atteint les $100 \%$ à la sortie de la station. Shuval et al [16] estiment la sédimentation des œufs d'Ascaris à $0,6 \mathrm{mh}^{-1}$ et $0,26 \mathrm{mh}^{-1}$ pour les œufs de Taenia dans les eaux au repos.

Si la décantation primaire semble permettre la sédimentation de la majorité de la charge en œufs d'Helminthes, il n'en n'est pas de même pour les kystes de protozoaires. Ces derniers se révèlent plus difficiles à éliminer. Le taux d'abattement des kystes observé après la décantation primaire est de $30 \%$. Cela peut être dû à leurs tailles nettement plus petites que les œufs. Notamment pour le kyste de Giardia spp (taille 10 à $13 \mu \mathrm{m}$ ), on enregistre un abattement de $6 \%$. Alors que les kystes d'Entamoeba coli et Entamoeba histolytica de plus grandes tailles, présentent des taux d'élimination respectivement de 43 et $54 \%$ au niveau de la décantation primaire. Nous avons constaté par ailleurs que le rendement d'élimination des kystes est d'environ $97 \%$ à la sortie de la station. Cette disparition quasi-totale des kystes est la résultante de deux phénomènes : la survie des kystes dans l'environnement et l'impact de traitement des eaux. D'un côté, les études de Shuval et al [16] sur des kystes de protozoaires montrent une survie de 15 à 30 jours dans les matières fécales ainsi que les eaux usées et de 10 à 15 jours sur les récoltes, de l'autre Schwartzbrod et al [6] prouvent que le traitement des eaux par des boues activées réduit à des taux de 84 à $99 \%$ les kystes de Giardia. Nous avons enregistré pour ce dernier un taux d'élimination de $98 \%$ dans la station de Cherguia.

En conclusion on peut constater l'efficacité du traitement d'épuration par boues activées puisque aussi bien pour les kystes de protozoaires que pour les œufs d'Helminthes, l'élimination est très importante avec un abattement allant de 97 à $100 \%$.

Toutefois il est important de signaler qu'en eau épurée $14 \%$ d'échantillons sont encore positifs en kystes de protozoaires avec des concentrations de $1,410^{1}$ kystes $\mathrm{l}^{-1}$.

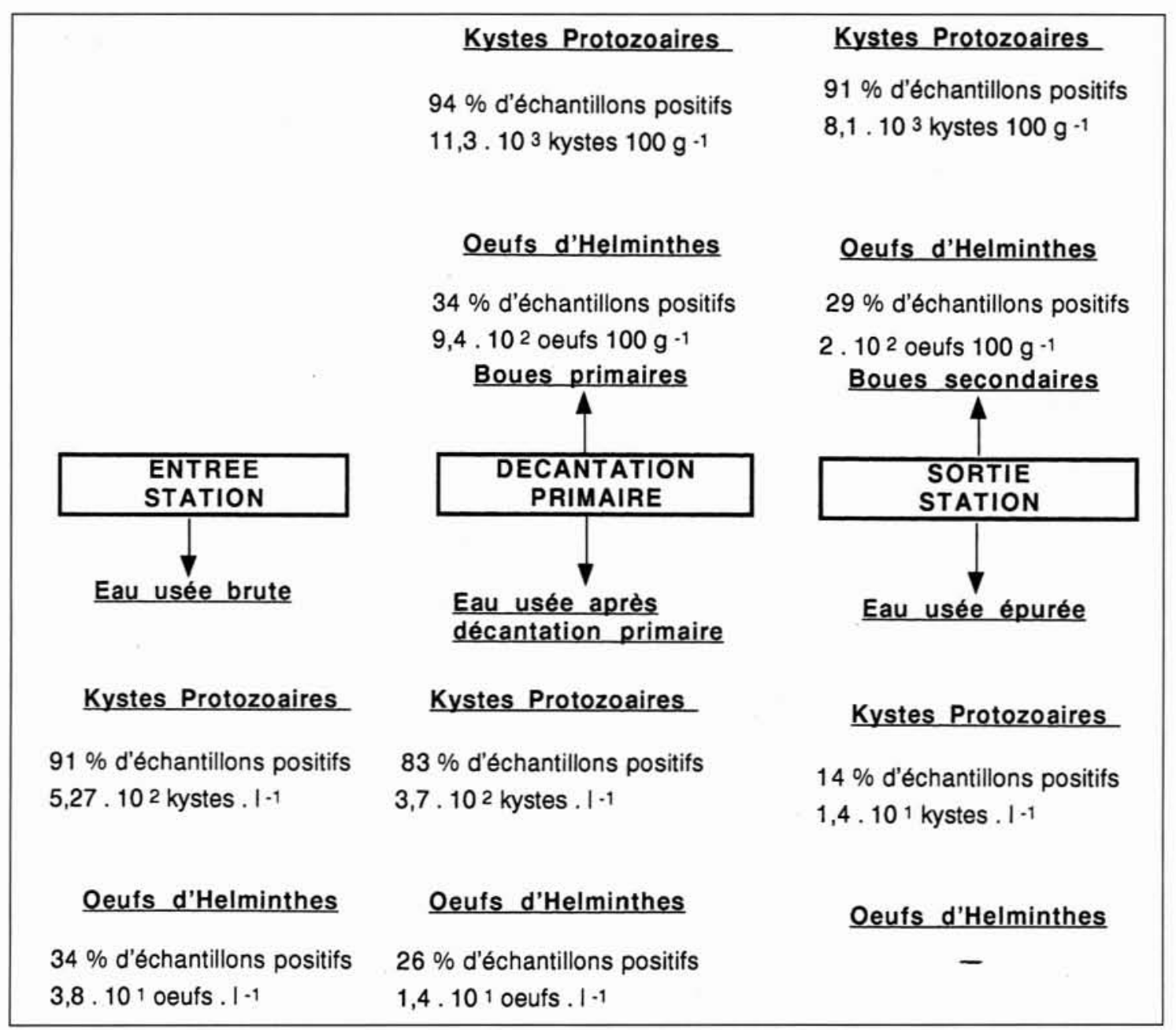

Schéma 1 : Devenir des æufs et kystes de parasites au cours du traitement d'épuration. 


\section{Eau : ressources, aménagements, environnement}

\section{RÉFÉRENCES}

[1] CHUTE G.C., SMITH R.P., BARON J.A., 1987. Risk Factor For endemic Giardiasis, Am. J.Publ. Healh. 77, pp 585-587.

[2] LAPHAM S.C., HOPKINS D.S. WITH M.C., BLAIR J.R., BISSELL R.A. SIMPSON G.L., 1987. A prospective Study of Giardiasis and water supplies in Colorado. Am.Journ Publ. Health, 77, pp 354-355.

[3] ECKERT J., 1993. Träger und Ausscheider von protozoen, Zbl. Hyg.. $194,173-185$.

[4] KENNOU M.F., 1983. Les parasitoses d'origine alimentaire en Tunisie. Arch. Inst. Pasteur Tunis, 60, (3-4), pp 393-407.

[5] ALOUINI Z., 1993. Flux de la charge parasitaire dans cinq stations d'épuration en Tunisie. Rev. Sci. de l'eau, 6, pp 453-462.

[6] SCHWARTZBROD J, STRAUS S.,1989. Devenir des kystes de Giardia au cours d'un cycle d'épuration. T.S.M., Nº, pp 331-334.

[7] STRAUSS M., 1985. Survival of excreted pathogens in excreta and faecal sludges. IRCWD NEWS, 23, pp 4-9.

[8] O.M.S. Rapport, 1989. Health guide lines for the use of waste water in agriculture and aquaculture. $N^{\circ} 778$, Geneve, Suisse.

[9] STIEN J.L., SCHWARTZBROD J., 1988. Flux d'œufs d'helminthes parasites dans les stations d'épuration par boues activées. T.S.M., $\mathrm{N}^{\circ} 10$, pp 495-498.
[10] PANICKER P.V.R.C., KRISHNAMOORTHI K.P., 1981. Parasite egg and cyst reduction in oxidation ditches and aerated lagoons. J. Wat Poll control Fed, 53, pp 1413-1419.

[11] FOX, J.C.FITZGERALD P.R., 1979. The presence of Giardia lamblia cysts in sewage sludges from the Chicago area, EPA 600/9 - $79-001$ pp 268-269.

[12] AKIN E. W., JAKUBOWSKI W., LUCAS J.B. PAHRENT H.R., 1978. Health hazards associated with waste water effluents and sludge : microbiological considerations. In Proceedings Conference San Antonio "Risk assesment and health effects of land application of municipal waste water and sludges", Sagik and Sorber ed, pp 9-26.

[13] JAKUBOWSKI W., ERICKSEN. H., 1979. Methods for detection of Giardia cysts in water supplies waterborne transmission of giardiasis, EPA 600/9 - 79 - 001, pp 193-210.

[14] BEN RACHID M.S., BEN SALEM M., 1968. Fréquence des helminthiases humaines en Tunisie. Archs. Inst. Pasteur, 45, pp139-151.

[15] PEPIN D., CAMBON M. COULET M., 1983. Etude parasitologique des boues résiduaires conditionnées thermiquement et chimiquement. J.Fr Hydrol., 14, pp 315-322

[16] SHUVAL M.I. ADIN A. FATAL B, RAWITZ E., YEKUTIEL P., 1986. Waste water irrigation in developping countries : Health effects and technical solutions' World Bank Technical, paper $\mathrm{N}^{\circ} 51$ pp 21-38. 